\title{
On the formation of convex and starlike functions with the form of Hadamard multiplication redefine
}

Ismet Yildiz

Department of Mathematics, Duzce University, Duzce, Turkey

Received: 24 October 2018, Accepted: 6 December 2018

Published online: 29 December 2018.

Abstract: In this study, some families of starlike and convex functions for which the image domain has special geometric property were considered in the study. The Hadamard product created by these functions has been formed and these functions has been redefined using Hadamard multiplication.

Keywords: Starlike functions, univalent functions, convex functions, Hadamard multiplication.

\section{Introduction}

First of all we begin our study with the definition of complex differentiable.

Definition 1. If function $f(z)=z+\sum_{k \geq 2} a_{k} z^{k}$ and function $g(z)=z+\sum_{k \geq 2} b_{k} z^{k}$ are analytic in $|z|<1$, where $f(0)=0$ and $f^{\prime}(0)=1, g(0)=0$ and $g^{\prime}(0)=1, f(z)$ and $g(z)$ are univalent function.

\section{Theorem 1.}

(i) If the function $f(z)$ and $g(z)$ are convex within the unit disk $|z|<1$. Then for each $\left|z_{0}\right|<1$

$$
z\left[\frac{f(z)-f\left(z_{0}\right)}{z-z_{0}}\right]^{2}
$$

is a function starlike.

(ii) If $\Re\left(\frac{z f^{\prime}(z)}{f(z)}\right)>\frac{1}{2}$. Then function $f(z)$ is graded $\frac{1}{2}$ starlike function in $|z|<1$ [4,5].

If $\Re\left(\frac{z g^{\prime}(z)}{g(z)}\right)>\frac{1}{2}$. Then function $g(z)$ is graded $\frac{1}{2}$ starlike function in $|z|<1[4,5]$.

Theorem 2. Provide the following conditon for the function $f(z)$ to be starlike the order $\frac{1}{2}$ of the starlike function

$$
\operatorname{Re}\left(\frac{f\left(z_{1}\right)-f\left(z_{2}\right)}{z_{1}-z_{2}} \frac{z_{2}}{f\left(z_{2}\right)}\right)>\frac{1}{2}
$$

where $\left|z_{1}\right|<1$ and $\left|z_{2}\right|<1$ [3].

Definition 2. The multiplication of analytic and univalent function $f(z)$ and $g(z)$ in the unit disk $|z|<1$ is defined as follows

$$
(f * g)(z)=z+\sum_{k \geq 2} a_{k} b_{k} z^{k}
$$


where $f(z)=z+\sum_{k \geq 2} a_{k} z^{k}$ and $g(z)=z+\sum_{k \geq 2} b_{k} z^{k}$. This is multiplication of geometric functions by the following.[1]

(i) If the function $f(z)$ and function $g(z)$ are convex. Then the product function $f(z) * g(z)$ is convex.

(ii) This multiplication function $f(z) * g(z)$ is univalent [2].

\section{Definition 3.}

(i) The function $f(z)$ is required and sufficient conditon to be a starlike function within the unit disk $|z|<1$.

$$
\operatorname{Re}\left(\frac{z f^{\prime}(z)}{f(z)}\right)>0
$$

(ii) The function $g(z)$ is required and sufficient condition to be a convex function within the unit disk $|z|<1$.

$$
\operatorname{Re}\left(1+\frac{z f^{\prime \prime}(z)}{f^{\prime}(z)}\right)>0,
$$

where $f^{\prime}(z) \neq 0$.

Lemma 1. If $f(z)$ is a function that has been normalized. Then

(i) If $\operatorname{Re}\left(1+\frac{z f^{\prime \prime}(z)}{f^{\prime}(z)}\right)>\alpha$ then function $f(z)$ for inequality is $\alpha$ grade convex function.

(ii) If $\Re\left(\frac{z f^{\prime}(z)}{f(z)}\right)>\alpha$ then function $f(z)$ for inequality is $\alpha$ grade starlike function. Where if $|z| \leq R<1$ and $f(0)=0$, $f^{\prime}(0)=1$. Then $f(z)$ is normalized. For each of these classes is to find a function $g(z)$ that satisfies the requirement for Hadamard multiplication.

Theorem 3. $F(z)$ and $G(z)$ functions get $\frac{1}{2}$ order of starlike. If these functions meet the following requirements, the multiplication is almost convex.

(i) $\mathfrak{R}\left(\frac{z f^{\prime}(z)}{F(z)}\right)>0$,

(ii) $\operatorname{Re}\left(\frac{z(G * z f)^{\prime}}{F * G}\right)>0$ where $|z|<1$.

Theorem 4. A function $f(z)$ is said to $b$ convex of order $\frac{1}{2}$. If and only if

(i) $\frac{1}{z}\left[f * \frac{z+\frac{u+\alpha}{1-\alpha} z^{2}}{(1-z)^{3}}\right] \neq 0$ where $|z| \leq R<1$.

(ii) A function $f(z)$ is to be starlike of order $\frac{1}{2}$. If and only if $\frac{1}{z}\left[f * \frac{z+\frac{u+2 \alpha-1}{2-2 \alpha} z^{2}}{(1-z)^{2}}\right] \neq 0$ where $|z| \leq R<1$ [6].

Theorem 5. A function $f(z)$ is to be convex of order $2^{-r}$. If and only if

$$
\frac{1}{z^{2}}\left[f * \frac{z+\frac{u+2^{-r}}{1-2^{-r}} z^{2}}{(1-z)^{3}}\right] \neq 0
$$

where $|z| \leq R<1,|u|=1, u=-1$ and $r$ is positive integer.

Proof. A necessary and sufficient condition for a function $f(z)$ to be convex of order $2^{-r}$ in $|z|<R$ in that

$$
\Re\left(\frac{z f^{\prime \prime}(z)}{f^{\prime}(z)}\right)>2^{-r}
$$

where $r$ is positive integer. When $z=0$ we easily see that $\frac{z f^{\prime \prime}(z)}{f^{\prime}(z)}=1$. In this case, inequality is equivalent to

$$
\frac{\frac{\left[z f^{\prime}(z)\right]^{\prime}}{f^{\prime}(z)}-2^{-r}}{1-2^{-r}} \neq \frac{u-1}{u+1} .
$$


Hence

$$
(1+u)\left[\left[z f^{\prime}(z)\right]^{\prime}+\left(1-2^{-r}\right)-u\right] f^{\prime}(z) \neq 0
$$

If we take $f(z)=z+\sum_{k \geq 2} a_{k} z^{k}$. Then

$$
\left[z f^{\prime}(z)\right]^{\prime}=1+\sum_{k \geq 2} k^{2} a_{k} z^{k-1}=f^{\prime}(z) *\left(\sum_{k=1} k z^{k-1}\right)=f^{\prime}(z) * \frac{1}{(1-z)^{2}}
$$

This last equality is to the left side of Theorem 4.

$f^{\prime}(z) *\left[\sum_{k=1}\left(1-2^{1-r}-u+(1+u) k\right)\right] z^{k-1}=f(z) *\left[\frac{1-2^{1-r}-u}{1-z}+\frac{1+u}{(1-z)^{2}}\right]=f^{\prime}(z) *\left[\frac{2-2^{1-r}+\left(u+2^{1-r}-1\right) z}{(1-z)^{2}}\right]$

Hence this is equivalent to

$$
\frac{1}{z}\left[z f^{\prime}(z) * \frac{z+\frac{u+2^{1-r}-1}{2-2^{1-r}} z^{2}}{(1-z)^{2}}\right] \neq 0
$$

Since $z f^{\prime}(z) * g(z)=f(z) * z g^{\prime}(z)$, we may rewrite express as follows

$$
\frac{1}{z}\left[f(z) * \frac{z+\frac{u+2^{-r}}{1-2^{-r}} z^{2}}{(1-z)^{3}}\right] \neq 0
$$

Theorem 6. A function $f(z)$ is said to be starlike of order $2^{-r}$ if and only if

$$
\frac{1}{z}\left[f(z) * \frac{z+\frac{u+2^{-r}-1}{2-2^{1-r}}}{(1-z)^{3}}\right] \neq 0
$$

where $|z| \leq R<1,|u|=1, u \neq-1$.

Proof. A necessary and sufficient condition for a function $f(z)$ to be convex of order $2^{-r}$ in $|z|<R$ is that $g(z)=\int_{0}^{z} \frac{f(\zeta)}{\zeta} d \zeta$ in starlike of order $2^{-r}$ in $|z|<R \leq 1$. Then we have

$$
\frac{1}{z}\left[g(z) * \frac{z+\frac{u+2^{-r}}{1-2^{-r}} z^{2}}{(1-z)^{3}}\right]=\frac{1}{z}\left[f(z) * \frac{z+\frac{u+2^{1-r}}{2-2^{1-r}} z^{2}}{(1-z)^{2}}\right] .
$$

\section{Competing interests}

The authors declare that they have no competing interests.

\section{Authors' contributions}

All authors have contributed to all parts of the article. All authors read and approved the final manuscript. 


\section{References}

[1] G.Polya, I. J. Schoenberg, Remarks on de la Vallée Poussin means and convex conformal maps of the circle, Pacific Journal of Mathematics, 8(1958), 295-334.

[2] T. J. Suffridge, Convolutions of convex functions, Journal of Mathematics and Mechanics, 15 (1966) 795-804.

[3] S. Rusceweyh, Sheil-Small T., Hadamard products of Schlicht functions and the Pólya-Schoenberg conjecture, Comment Math. Helv. 48(1973) 119-135.

[4] T. J. Suffridge, Some remarks on convex maps of the unit disk, Duke Mathematical Journal, 37 (1970) 775-777.

[5] T. Sheil-Small, On convex univalent functions, Journal of the London Mathematical Society 1 (1969), 483-492.

[6] H. Silverman,E.M.Silvia, D.Telage, Convolution conditions for convexity, starlikeness and spiral-likeness, Mathematische Zeitschrift (1978) 125-130.

[7] S. Ruscheweyh, Linear operators between classes of prestarlike functions, Commentarii Mathematici Helvetici, 52(1), (1977), 497-509.

[8] S. Ruscheweyh, New criteria for univalent functions, Proc. Amer. Math. Soc. 49 (1975), 109-115.

[9] S. Owa, S. Fuku1, K. Sakaguch1, S. Ogawa, An application of the Ruscheweyh derivatives, International Journal of Mathematics and Mathematical Sciences, 9(4) (1986) 721-730. 